Volume 134, Number 1, Pages 39-50

S 0002-9939(05)07955-4

Article electronically published on June 2, 2005

\title{
SKEW CATEGORY, GALOIS COVERING AND SMASH PRODUCT OF A $k$-CATEGORY
}

\author{
CLAUDE CIBILS AND EDUARDO N. MARCOS
}

(Communicated by Martin Lorenz)

\begin{abstract}
In this paper we consider categories over a commutative ring provided either with a free action or with a grading of a not necessarily finite group. We define the smash product category and the skew category and we show that these constructions agree with the usual ones for algebras. In the case of the smash product for an infinite group our construction specialized for a ring agrees with M. Beattie's construction of a ring with local units. We recover in a categorical generalized setting the Duality Theorems of M. Cohen and S. Montgomery (1984), and we provide a unification with the results on coverings of quivers and relations by E. Green (1983). We obtain a confirmation in a quiver and relations-free categorical setting that both constructions are mutual inverses, namely the quotient of a free action category and the smash product of a graded category. Finally we describe functorial relations between the representation theories of a category and of a Galois cover of it.
\end{abstract}

\section{INTRODUCTION}

Throughout this paper we will consider small categories $\mathcal{C}$ over a commutative ring $k$, which means that the objects $\mathcal{C}_{0}$ form a set, the morphism set ${ }_{y} \mathcal{C}_{x}$ from an object $x$ to an object $y$ is a $k$-module and the composition of morphisms is $k$-bilinear.

We consider in addition $G$-categories and $G$-graded categories over $k$ where $G$ is a group, inspired by the work of $[8,12,9,19,14,7]$ and by a preliminary version of [13. A $G$-category over $k$ is provided by a category $\mathcal{C}$ with first a set action of $G$ on the objects and secondly $k$-module maps $s:{ }_{y} \mathcal{C}_{x} \rightarrow{ }_{s y} \mathcal{C}_{s x}$ for each $s \in G$ and for each couple of objects $x$ and $y$, verifying $s(g f)=(s g)(s f)$ in case $g$ and $f$ are morphisms which can be composed in the category. Moreover for elements $t, s$ in the group and a morphism $f$ we have $(t s) f=t(s f)$; we also require $1 f=f$ where 1 is the neutral element of $G$. In other words there is a group homomorphism from $G$ to the group of autofunctors of the category. If the action of $G$ is free on $\mathcal{C}_{0}$ we say that $\mathcal{C}$ is a free $G$-category.

Received by the editors December 22, 2003 and, in revised form, August 26, 2004.

2000 Mathematics Subject Classification. Primary 18A32, 16S35, 16G20.

The second author thanks CNPq (Brazil) for financial support, in the form of a productivity scholarship. The authors thank the IME of the Universidade de São Paulo for support during the preparation of this work.

(C)2005 American Mathematical Society 
A $G$-graded category $\mathcal{C}$ over $k$ has a direct sum decomposition of $k$-modules ${ }_{y} \mathcal{C}_{x}=\bigoplus_{s \in G}{ }_{y} \mathcal{C}_{x}{ }^{s}$ for each couple of objects, verifying ${ }_{z} \mathcal{C}_{y}{ }^{t}{ }_{y} \mathcal{C}_{x}{ }^{s} \subset{ }_{y} \mathcal{C}_{x}{ }^{t s}$ and ${ }_{x} 1_{x} \in{ }_{x} \mathcal{C}_{x}{ }^{1}$.

In section 2 we recall the definition of the quotient category $\mathcal{C} / G$, which makes sense only if $\mathcal{C}$ is a free $G$-category. It has been introduced and considered in [20, 5] as well as in subsequent work by several authors and recently in [7. Note that by definition Galois coverings are precisely given this way; namely, $\mathcal{C}$ is a Galois covering of the quotient $\mathcal{C} / G$. Next we consider the skew-category $\mathcal{C}[G]$, which is an analogue of the skew-ring construction. We prove a coherence result between our approach and the ring-theoretical construction in the case that $G$ is finite and the category has a finite number of objects. The main result in this section is that $\mathcal{C} / G$ and $\mathcal{C}[G]$ are equivalent.

In section 3 we consider a $G$-graded category $\mathcal{B}$ over $k$ and we define the smash product category $\mathcal{B} \# G$. In case $G$ is finite, we obtain again a coherence result with the $k$-algebra case used in [8]. The categorical approach for the smash product that we introduce here is equivalent to the ring without identity construction obtained by M. Beattie in [3]. Therefore the same remarks from the introduction of $[3$ are in force: the rings considered in [15, 18, differ from the present approach. Note that in the context of quivers and relations (that is, categories given through a presentation) the constructions described in [12] correspond to the smash product introduced in this paper.

The first result in section 3 relates the categorical smash product with quotients: if $\mathcal{B}$ is a $G$-graded category over $k$, then $\mathcal{B} \# G$ is a free $G$-category and there is a canonical isomorphism $(\mathcal{B} \# G) / G \simeq \mathcal{B}$. The second result of this section concerns a free $G$-category $\mathcal{C}$ over $k$ : the quotient category $\mathcal{C} / G$ is graded and the smash product $(\mathcal{C} / G) \# G$ is isomorphic to $\mathcal{C}$. As an immediate corollary we obtain that $(B \# G)[G]$ is equivalent to $\mathcal{B}$. We illustrate these results by considering the Kronecker category.

The combination of the quoted results provides a generalization of the CohenMontgomery Duality Theorems [8] in the version provided by M. Beattie [4, for a nonnecessarily finite group and a category with possibly infinitely many objects.

In 12 E. Green considers pairs $(\Gamma, \rho)$ formed by a locally finite, path connected directed graph $\Gamma$ and a set $\rho$ of relations, namely $k$-linear combinations of paths of $\Gamma$. This data presents a category over $k$ : first consider the free category on the set of paths, where objects are vertices and morphisms between two vertices are free $k$-modules having a basis given by the paths between these vertices. Secondly perform the quotient by the two-sided categorical ideal generated by $\rho$. Regular coverings considered in [12] concern categories over $k$ given by a fixed presentation which is locally finite. We consider categories over $k$ with no need of a specific presentation. In this context Galois coverings correspond to regular ones, see for instance [21.

We provide in this paper a confirmation in a uniform pure categorical setting that gradings of a category over $k$ are in one-to-one correspondence with Galois coverings, after the work of E. Green 12 .

In order to make precise the link with 8 we specialize our results to the case of a finite group $G$. Let $A$ be a $G$-graded $k$-algebra, which we consider as a single object category $\mathcal{C}_{A}$ with endomorphism ring $A$. The smash product algebra $A \# G$ has a free $G$-action and the corresponding skew group algebra $(A \# G)[G]$ is proved 
in $[8$ to be a matrix algebra over $A$ of size $|G|$. Now the smash product category $\mathcal{C}_{A} \# G$ we consider in this paper has $G$ as its set of objects. Since we assume here that $G$ is finite, we can consider the $k$-algebra of the category $a\left(\mathcal{C}_{A} \# G\right)$, namely the direct sum of all the $k$-module morphisms of $\mathcal{C}_{A} \# G$ equipped with the usual matrix product. We show that this algebra is precisely $A \# G$.

Our results in this specific case provide the existence of a single object full subcategory of $\mathcal{C}_{A} \# G[G]$ which is equivalent to the ambient one and isomorphic to $\mathcal{C}_{A}$. Since $\mathcal{C}_{A} \# G$ has precisely $|G|$ objects we infer the Cohen-Montgomery result quoted above.

In another direction note that Hochschild-Mitchell cohomologies of equivalent $k$-categories remain isomorphic through the corresponding change of bimodules. In [7. a Cartan-Leray spectral sequence is obtained relating the Hochschild-Mitchell cohomologies of the categories of a Galois covering $\mathcal{C} \rightarrow \mathcal{C} / G$. Our results show that this Cartan-Leray spectral sequence translates into a spectral sequence relating the Hochschild-Mitchell cohomologies of $\mathcal{C}$ and of $\mathcal{C}[G]$, since $\mathcal{C}[G]$ is equivalent to $\mathcal{C} / G$.

Finally in the last section we consider left modules over a category $\mathcal{C}$, namely contravariant functors from $\mathcal{C}$ to the category of $k$-modules. Note that if a category $\mathcal{C}$ over $k$ is presented by a quiver with relations, left $\mathcal{C}$-modules are precisely representations of the quiver subjected to the relations. In the case of a Galois covering of categories $\mathcal{C} \rightarrow \mathcal{B}$, modules over the base category $\mathcal{B}$ coincide with the fixed modules over the covering category under the action of the group $G$, while all the modules over the covering category coincide with graded ones over the base category. Note that in the case of rings, graded modules have been generalized to modules graded by a $G$-set and general versions of the Duality Theorems of M. Cohen and S. Montgomery have been obtained; see [16, 17, 10].

\section{GALOIS COVERINGS AND THE SKEW CATEGORY}

Let $\mathcal{C}$ be a free $G$-category over a commutative ring $k$. Recall that the $G$-set of objects $\mathcal{C}_{0}$ is free; namely, if $s x=x$ for an object $x$, then $s=1$.

Definition 2.1. Given a free $G$-category $\mathcal{C}$ over $k$, the quotient category $\mathcal{C} / G$ is the category over $k$ whose objects are the $G$-orbits of $\mathcal{C}_{0}$. For two orbits $\alpha$ and $\beta$ the $k$-module of morphisms from $\alpha$ to $\beta$ is

$$
{ }_{\beta}(\mathcal{C} / G)_{\alpha}=\left(\bigoplus_{x \in \alpha, y \in \beta}{ }_{y} \mathcal{C}_{x}\right) / G
$$

Note that $\bigoplus_{x \in \alpha, y \in \beta}{ }_{y} \mathcal{C}_{x}$ is a $k G$-module and $\left(\bigoplus_{x \in \alpha, y \in \beta}{ }_{y} \mathcal{C}_{x}\right) / G$ is the largest $k$ module quotient with trivial $G$-action: if $X$ is a $k G$-module, then $X / G=X /(\operatorname{ker} \epsilon) X$ where $\epsilon: k G \rightarrow k$ is the augmentation map, i.e. the $k$-linear map given by $\epsilon(s)=1$ for all $s \in G$.

Composition in $\mathcal{C} / G$ is deduced from composition in $\mathcal{C}$ and is well defined precisely because the action of $G$ on the objects is free.

By definition a Galois covering is the projection functor from a free $G$-category to its quotient. 
Lemma 2.2. Let $\mathcal{C}$ be a free $G$-category over $k$, let $\alpha$ and $\beta$ be orbits of objects and let $x_{\alpha} \in \alpha$ and $x_{\beta} \in \beta$ be representatives. Then ${ }_{\beta}(\mathcal{C} / G)_{\alpha}$ and $\bigoplus_{s \in G} x_{\beta} \mathcal{C}_{s x_{\alpha}}$ are canonically isomorphic.

Proof. Consider the normalization map

$$
\bigoplus_{x \in \alpha, y \in \beta}{ }_{y} \mathcal{C}_{x} \stackrel{\phi}{\longrightarrow} \bigoplus_{s \in G}{ }_{x_{\beta}} \mathcal{C}_{s x_{\alpha}}
$$

given by $\phi\left({ }_{y} f_{x}\right)=s f$ where $s$ is the unique group element such that $s y=x_{\beta}$. Note that $\phi(t f)=\phi(f)$; hence $\phi:{ }_{\beta}(\mathcal{C} / G)_{\alpha} \longrightarrow \bigoplus_{s \in G} x_{\beta} \mathcal{C}_{s x_{\alpha}}$ is well defined. Conversely, consider

$$
\psi: \bigoplus_{s \in G}{ }_{x_{\beta}} \mathcal{C}_{s x_{\alpha}} \hookrightarrow \bigoplus_{x \in \alpha, y \in \beta}{ }_{y} \mathcal{C}_{x} \rightarrow{ }_{\beta}(\mathcal{C} / G)_{\alpha}
$$

which is an inverse for $\phi$.

Definition 2.3. Let $\mathcal{C}$ be a $G$-category over $k$. The skew category $\mathcal{C}[G]$ has a set of vertices $(\mathcal{C}[G])_{0}=\mathcal{C}_{0}$ and morphisms ${ }_{y}(\mathcal{C}[G])_{x}=\bigoplus_{s \in G}{ }_{y} \mathcal{C}_{s x}$.

Composition of morphisms is provided by the composition of $\mathcal{C}$ after adjustment. In order to make this precise one needs to keep track of the component where a morphism is located: we put $f=f_{s}$ in case $f \in{ }_{y} \mathcal{C}_{s x}$. This way $f_{s} \neq f_{s^{\prime}}$ for $s \neq s^{\prime}$ in case $s x=s^{\prime} x$. Let $g=g_{t} \in{ }_{z} \mathcal{C}_{t y}$ and $f=f_{s} \in{ }_{y} \mathcal{C}_{s x}$. Then $g f=g \circ(t f)$.

We show now a coherence result with the usual skew-algebra construction.

Proposition 2.4. Let $G$ be a finite group and let $\mathcal{C}$ be a $G$-category over $k$ with a finite number of objects. Let $a(\mathcal{C})$ be the $k$-algebra associated to $\mathcal{C}$, namely $a(\mathcal{C})=\bigoplus_{x, y \in \mathcal{C}_{0}}\left({ }_{y} \mathcal{C}_{x}\right)$ provided with the matrix product induced by the composition of morphisms.

Then $G$ acts on $a(\mathcal{C})$ by algebra automorphisms and $a(\mathcal{C}[G])$ is the skew algebra $a(\mathcal{C})[G]$.

Proof. Recall that if $A$ is a $k$-algebra with $G$ acting by automorphisms on it, the skew group algebra $A[G]$ is the $k$-module $A \otimes k G$ with the twisted product provided by $s a=s(a) s$ for all $s \in G$ and $a \in A$. As is well known and easy to check the twisting (see for instance [6]) conditions are satisfied and the skew group algebra is a $k$-algebra which has $A$ and $k G$ as subalgebras.

The isomorphism of algebras $\psi: a(\mathcal{C}[G]) \rightarrow a(\mathcal{C})[G]$ is defined as follows. Let $f_{s} \in{ }_{y} \mathcal{C}_{s x}$ be an elementary morphism of ${ }_{y} \mathcal{C}[G]_{x}$. Then $\psi\left(f_{s}\right)=f \otimes s$. We have that

while

$$
\psi(g f)=\psi(g \circ(t f))=g(t f) \otimes t s
$$

Moreover

$$
\psi(g) \psi(f)=(g \otimes t)(f \otimes s)=g(t f) \otimes t s .
$$

$$
\psi(1)=\psi\left(\sum{ }_{x} 1_{x}\right)=\sum \psi\left({ }_{x} 1_{x}\right)=\sum\left({ }_{x} 1_{x} \otimes 1_{G}\right)=\left(\sum{ }_{x} 1_{x}\right) \otimes 1_{G}=1 \otimes 1_{G} .
$$

The following result shows a fact that we need to be true in $\mathcal{C}[G]$. Together with the coherence property above, it justifies the definition we gave for the skew category $\mathcal{C}[G]$. 
Lemma 2.5. Let $\mathcal{C}$ be a $G$-category over $k$. Objects in the same $G$ orbit are isomorphic in $\mathcal{C}[G]$.

Proof. If $s x=y$, then ${ }_{y} 1_{y} \in{ }_{y} \mathcal{C}[G]_{x}$ and ${ }_{x} 1_{x} \in{ }_{x} \mathcal{C}[G]_{y}$. These morphisms are mutual inverses.

Proposition 2.6. Let $\mathcal{C}$ be a $G$-category over $k$ and let $\mathcal{C}[G]$ be the skew category. Choose an element $x_{\alpha}$ in each orbit $\alpha$ and let $\mathcal{C}[G]$ be the full subcategory of $\mathcal{C}[G]$ given by these objects. Then the categories $\mathcal{C}[G]$ and $\underline{\mathcal{C}}[G]$ are equivalent.

Proof. Consider the functor $F: \mathcal{C}[G] \rightarrow \mathcal{C}[G]$ defined on objects by $F(x)=x_{\alpha}$ if the orbit of $x$ is $\alpha$. Since $x$ and $x_{\alpha}$ are canonically isomorphic (see the Lemma above) a morphism $f: x \rightarrow y$ provides a unique morphism $x_{\alpha} \rightarrow x_{\beta}$ where $\beta$ is the orbit of $y$. This construction is functorial; moreover $F$ and the natural embedding are inverse equivalences.

Proposition 2.7. Let $\mathcal{C}$ be a free $G$-action category over $k$. Then the categories $\mathcal{C} / G$ and $\underline{\mathcal{C}[G]}$ are isomorphic.

Proof. This is a consequence of Lemma 2.2.

We have proved the following.

Theorem 2.8. Let $\mathcal{C}$ be a free $G$-category over $k$. The quotient category $\mathcal{C} / G$ and the skew category $\mathcal{C}[G]$ are equivalent.

Remark 2.9. This theorem suggests that the categories $\mathcal{C}[G]$ or $\mathcal{C}[G]$ can be considered as a substitute for the quotient category in case the action of $G$ on $\mathcal{C}$ is not free.

\section{SMASh PRODUCT CATEGORY}

Let $G$ be a group. A $G$-graded category $\mathcal{B}$ over $k$ is a category over $k$ together with a decomposition of each $k$-module of morphisms ${ }_{y} \mathcal{B}_{x}=\bigoplus_{s \in G}{ }_{y} \mathcal{B}_{x}{ }^{s}$ such that ${ }_{z} \mathcal{B}_{y}{ }^{t}{ }_{y} \mathcal{B}_{x}{ }^{s} \subset{ }_{z} \mathcal{B}_{x}{ }^{t s}$. In particular ${ }_{x} \mathcal{B}_{x}$ is a $G$-graded algebra for each object $x$.

The smash product category $\mathcal{B} \# G$ is defined below. We will show a coherence result in case $\mathcal{B}$ is a single object category given by a $G$-graded $k$-algebra. Then we will prove that a Galois covering of categories is isomorphic to a smash product of categories.

Definition 3.1. The smash product category $\mathcal{B} \# G$ has object set $\mathcal{B}_{0} \times G$. Let $(x, s)$ and $(y, t)$ be objects. The $k$-module of morphisms is defined as follows:

$$
{ }_{(y, t)}(\mathcal{B} \# G)_{(x, s)}={ }_{y} \mathcal{B}_{x} t^{-1} s .
$$

Note that ${ }_{(y, t)}(\mathcal{B} \# G)_{(x, s)}$ and ${ }_{(y, u t)}(\mathcal{B} \# G)_{(x, u s)}$ are $k$-module morphisms from different objects which coincide as $k$-modules.

In order to define the composition map

$$
{ }_{(z, u)}(\mathcal{B} \# G)_{(y, t)} \otimes_{k}{ }_{(y, t)}(\mathcal{B} \# G)_{(x, s)} \longrightarrow{ }_{(z, u)}(\mathcal{B} \# G)_{(x, s)}
$$

note that the left-hand side is

$$
{ }_{z} \mathcal{B}_{y}^{u^{-1} t} \otimes_{k}{ }_{y} \mathcal{B}_{x} t^{-1} x
$$

while the right-hand side is ${ }_{z} \mathcal{B}_{x}{ }^{u^{-1} s}$. The graded composition of $\mathcal{B}$ provides the required map. 
Proposition 3.2. Let $\mathcal{B}$ be a $G$-graded category over $k$. The category $\mathcal{B} \# G$ is a free $G$-category and $(\mathcal{B} \# G) / G=\mathcal{B}$.

Proof. On objects we define $u(x, s)=(x, u s)$.

Let $f$ be a morphism in ${ }_{(y, t)}(\mathcal{B} \# G)_{(x, s)}={ }_{y} \mathcal{B}_{x}^{t^{-1}}$. We define

$$
u f=f \in{ }_{(y, u t)}(\mathcal{B} \# G)_{(x, u s)} .
$$

In other words the action is obtained by translation. Clearly this is an action and moreover the action on objects is free. Note that orbits of objects are in one-to-one correspondence with objects of $\mathcal{B}$, by retaining the first component of each couple.

This shows that $[(\mathcal{B} \# G) / G]_{0} \equiv \mathcal{B}_{0}$. Moreover recall that by definition we have

$$
{ }_{y}[(\mathcal{B} \# G) / G]_{x}=\left[\bigoplus_{s \in G, t \in G}(y, t)(\mathcal{B} \# G)_{(x, s)}\right] / G .
$$

The action of $G$ on the numerator transports ${ }_{(y, 1)}(\mathcal{B} \# G)_{(x, s)}$ to ${ }_{(y, u)}(\mathcal{B} \# G)_{(x, u s)}$. Consequently those $k$-modules are identified in the quotient. This remark shows the following:

$$
\left[\bigoplus_{s \in G, t \in G}(y, t)(\mathcal{B} \# G)_{(x, s)}\right] / G=\bigoplus_{s \in G}(y, 1)(\mathcal{B} \# G)_{(x, s)}=\bigoplus_{s \in G}{ }_{y} \mathcal{B}_{x}{ }^{s} .
$$

The following is a natural consequence of the former proposition.

Corollary 3.3. Let $G$ be a group and $\mathcal{B}$ be a $G$-graded category over $k$. Then the categories $(B \# G)[G]$ and $\mathcal{B}$ are equivalent.

Proof. We know that $\mathcal{B} \# G$ is a Galois covering of $\mathcal{B}$. The result of the previous section shows that $\mathcal{B}[G]$ is equivalent to $\mathcal{B} / G$; namely, $(\mathcal{B} \# G)[G]$ is equivalent to $(\mathcal{B} \# G) / G=\mathcal{B}$.

Remark 3.4. In case a ring $A$ is graded by a finite group the coherence result below shows that the preceding corollary is the Cohen-Montgomery Duality Theorem (see [8] and [11). Note that using rings with local units, the Duality Theorem is still valid even if $G$ is not finite by the results of [4, 2]; see also [1].

Remark 3.5. In the case of an infinite group grading of a $k$-algebra $A$ the preceding corollary shows that the category $\left(\mathcal{C}_{A} \# G\right)[G]$ is equivalent to $\mathcal{C}_{A}$, where $\mathcal{C}_{A}$ is the single object category over $k$ determined by $A$.

In order to prove the coherence property of the categorical smash product with the smash product of a graded $G$-algebra we first recall its definition. Note that the usual smash product of a graded algebra requires $G$ to be finite; see for instance 8 . As quoted in the Introduction, the categorical smash product we consider in this paper is an alternative approach to the ring without identity defined by M. Beattie in [3].

Definition 3.6. Let $A$ be a $G$-graded $k$-algebra where $G$ is a finite group. The smash product $A \# k^{G}$ is the twisted (see for instance [6]) tensor product of algebras $A \otimes k^{G}$ where the twist map $\tau: k^{G} \otimes A \rightarrow A \otimes k^{G}$ is given as follows. Let $\delta_{s}$ be the Dirac mass on an element $s \in G$; namely, $\delta_{s}$ is the set map from $G$ to $k$ assigning 0 
to every element except $s$, while the value on $s$ is 1 . Let $f_{t}$ be an element of degree $t$ in $A$. Then

$$
\tau\left(\delta_{t} \otimes f_{s}\right)=f_{s} \otimes \delta_{s^{-1} t} .
$$

The verifications of the twisting properties of $\tau$ insuring associativity are well known. We provide them for the convenience of the reader and in order to avoid any missprint for $\tau$. We omit the tensor product symbol as well as the map $\tau$; in other words, we consider $\delta_{t} f_{s}=f_{t} \delta_{s^{-1} t}$ as the product in $A \otimes k^{G}$.

First observe that

$$
\left(\delta_{u} \delta_{t}\right) f_{s}=0 \text { if } u \neq t \text { while }\left(\delta_{u} \delta_{t}\right) f_{s}=f_{s} \delta_{s^{-1} t} \text { if } u=t
$$

and

$\delta_{u}\left(\delta_{t} f_{s}\right)=\delta_{u} f_{s} \delta_{s^{-1} t}=f_{s} \delta_{s^{-1} u} \delta_{s^{-1} t}=0$ if $u \neq t$ while $\delta_{u}\left(\delta_{t} f_{s}\right)=f_{s} \delta_{s^{-1} t}$ if $u=t$.

Second

Moreover

$$
\begin{gathered}
\delta_{u}\left(g_{t} f_{s}\right)=g_{t} f_{s} \delta_{(t s)^{-1} u} \text { since } g_{t} f_{s} \in A_{t s} \\
\left(\delta_{u} g_{t}\right) f_{s}=g_{t} \delta_{t^{-1} u} f_{s}=g_{t} f_{s} \delta_{s^{-1} t^{-1} u} .
\end{gathered}
$$

$$
1_{k^{G}} f_{s}=\sum_{u \in G} \delta_{u} f_{s}=\sum_{s \in G} f_{s} \delta_{s^{-1} u}=f_{s} 1_{k^{G}} .
$$

Recall that in case $\mathcal{B}$ is a finite object set category over $k$, the $k$-algebra of morphisms of $\mathcal{B}$ is denoted $a(\mathcal{B})$ : it is the direct sum of the morphism $k$-modules of $\mathcal{B}$ equipped with the matrix product.

Proposition 3.7. Let $G$ be a finite group and let $A$ be a G-graded k-algebra. Let $\mathcal{B}_{A}$ be the single object category over $k$ with endomorphism $k$-algebra $A$. Then the category $\mathcal{B}_{A}$ is $G$-graded and $a\left(\mathcal{B}_{A} \# G\right)=A \# G$.

Note that if $G$ is not finite $a\left(\mathcal{B}_{A} \# G\right)$ can still be considered (see [4, 17, 10]) as a ring with a set of local units; the above proposition can also be proved in this more general context where $A \# G$ is defined as a ring of this sort.

Proof. We define a map $\phi: a\left(\mathcal{B}_{A} \# G\right) \longrightarrow A \# G$ as follows. Consider an elementary matrix ${ }_{t} F_{s} \in a\left(\mathcal{B}_{A} \# G\right)$ with all entries zero except perhaps the entry $\left(x_{0}, t\right)-\left(x_{0}, s\right)$ with value an element $f_{t^{-1} s} \in{ }_{\left(x_{0}, t\right)}\left(\mathcal{B}_{A} \# G\right)_{\left(x_{0}, s\right)}$. Observe that

$$
{ }_{\left(x_{0}, t\right)}\left(\mathcal{B}_{A} \# G\right)_{\left(x_{0}, s\right)}={ }_{x_{0}}\left(\mathcal{B}_{A}\right)_{x_{0}}{ }^{t^{-1} s}=A_{t^{-1} s} .
$$

We put

$$
\phi\left({ }_{t} F_{s}\right)=f_{t^{-1} s} \delta_{s^{-1}}
$$

Note first that the unit element in $a(\mathcal{B} \# G)$ is $1=\sum_{s \in G} s 1_{s}$ where ${ }_{s} 1_{s}$ is the elementary matrix with nonzero entry only on the entry $\left(x_{0}, s\right)-\left(x_{0}, s\right)$ with value $1 \in A_{s^{-1} s}=A_{1}$. Then

$$
\phi\left(\sum_{s \in G}{ }_{s} 1_{s}\right)=\sum_{s \in G} 1_{1} \delta_{s^{-1}}=1_{A \# G} .
$$

Let ${ }_{v} G_{u}$ and ${ }_{t} F_{s}$ be elementary matrices. Then ${ }_{v} G_{u} F_{s}=0$ if $u \neq t$ and ${ }_{v} G_{u}{ }_{u} F_{s}$ is the elementary matrix ${ }_{v}(G F)_{s}$ with zero entries except a possible nonzero entry $\left(x_{0}, v\right)-\left(x_{0}, s\right)$ with value $g_{v^{-1} t} f_{t^{-1} s}$. We have that

$$
\phi\left({ }_{v} G_{u}\right) \phi\left({ }_{t} F_{s}\right)=g_{v^{-1} u} \delta_{u^{-1}} f_{t^{-1} s} \delta_{s^{-1}}=g_{v^{-1} u} f_{t^{-1} s} \delta_{s^{-1} t u^{-1}} \delta_{s^{-1}} .
$$


Note that if $u \neq t$ we have $\phi\left({ }_{v} G_{u}\right) \phi\left({ }_{t} F_{s}\right)=0$ while if $u=t$,

$$
\phi\left({ }_{v} G_{u}\right) \phi\left({ }_{t} F_{s}\right)=g_{v^{-1} u} f_{t^{-1} s} \delta_{s^{-1}}=\phi\left({ }_{v} G_{t}{ }_{t} F_{s}\right) .
$$

Clearly the morphism we have defined is an injective and surjective $k$-module map, since the image of the elementary matrix ${ }_{t} F_{s}$ belongs to $A_{t^{-1} s} \otimes \delta_{s^{-1}}$.

The following result can be linked with Proposition 3.2. in order to obtain that the categorical smash product and the categorical quotient are inverse constructions between graded categories and free action categories.

Theorem 3.8. Let $G$ be a group and let $\mathcal{C}$ be a free $G$-category over $k$. Then $\mathcal{C} / G$ is $G$-graded and the category $(\mathcal{C} / G) \# G$ is isomorphic to $\mathcal{C}$.

Proof. Let $\left\{x_{\alpha} \mid x_{\alpha} \in \alpha\right\}$ be a choice of representatives in each $G$-orbit of objects of $\mathcal{C}$. Recall that ${ }_{\beta}(\mathcal{C} / G)_{\alpha}=\left[\bigoplus_{x \in \alpha, y \in \beta}{ }_{y} \mathcal{C}_{x}\right] / G$. Let ${ }_{y} f_{x} \in{ }_{y} \mathcal{C}_{x}$. In order to define the degree $\operatorname{deg}\left[{ }_{y} f_{x}\right]$ of the class $\left[{ }_{y} f_{x}\right]$ we first normalize ${ }_{y} f_{x}$ in order to obtain as source object $x_{\alpha}$, and then we retain the gap between the target object obtained and $x_{\beta}$. More precisely there is a unique $s \in G$ such that $s x=x_{\alpha}$. Then there is a unique element of $G$ that we denote $(\operatorname{deg}[f])^{-1}$ such that $(\operatorname{deg}[f])^{-1} x_{\beta}=s y$.

First we note that $\operatorname{deg}[f]$ is well defined: let $u \in G$; then $u f \in{ }_{u y} \mathcal{C}_{u x}$. The normalization uses $s u^{-1}$ and the target object is again $s y$.

Second we define

$$
{ }_{\beta}(\mathcal{C} / G)_{\alpha}{ }^{s}=\left\{\sum[f] \mid f \in{ }_{y} \mathcal{C}_{x} \text { where } x \in \alpha, y \in \beta \text { and } \operatorname{deg}[f]=s\right\} .
$$

Then ${ }_{\beta}(\mathcal{C} / G)_{\alpha}=\bigoplus_{s \in G}(\mathcal{C} / G)_{\alpha}{ }^{s}$.

Finally we check that $\operatorname{deg}(g f)=\operatorname{deg}(g) \operatorname{deg}(f)$. Indeed consider $(\operatorname{deg} g)^{-1} x_{\gamma} g_{x_{\beta}}$ and $(\operatorname{deg} f)^{-1} x_{\beta} f_{x_{\alpha}}$. In order to compose those morphisms we first have to adjust $g$ in order that its source object coincides with the target object of $f$. The resulting composition is

$$
(\operatorname{deg} f)^{-1}(\operatorname{deg} g)^{-1} x_{\gamma}\left[\left(\operatorname{deg} f^{-1}\right) g f\right]_{x_{\alpha}} .
$$

Hence $(\operatorname{deg}(g f))^{-1}=(\operatorname{deg} f)^{-1}(\operatorname{deg} g)^{-1}$ and $\operatorname{deg}(g f)=(\operatorname{deg} g)(\operatorname{deg} f)$.

Using the graduation we have just described we define a functor

$$
F:(\mathcal{C} / G) \# G \rightarrow \mathcal{C}
$$

as follows. Let $(\alpha, s)$ be an object of the smash category. Then $F(\alpha, s)=s x_{\alpha}$.

Recall that by definition

$$
{ }_{(\beta, t)}(\mathcal{C} / G)_{(\alpha, s)}={ }_{\beta}(\mathcal{C} / G)_{\alpha}{ }^{t^{-1} s} .
$$

Let $\left.{ }_{s^{-1} t x_{\beta}}[f]_{x_{\alpha}} \in{ }_{(\beta, t)}(\mathcal{C} / G) \# G\right)_{(\alpha, s)}$ where $f \in{ }_{s^{-1} t x_{\beta}}(\mathcal{C})_{x_{\alpha}}$. We define

$$
F\left({ }_{s^{-1} t x_{\beta}}[f]_{x_{\alpha}}\right)=s f \in{ }_{t x_{\beta}} \mathcal{C}_{s x_{\alpha}} .
$$

We consider now

$$
t^{-1} u x_{\gamma}[g]_{x_{\beta}} \in{ }_{(\gamma, u)}(\mathcal{C} \# G)_{(\beta, t)} .
$$

In order to compose $[g]$ and $[f]$ in $(\mathcal{C} / G) \# G$ we have to adjust $g$ using the $G$-action, obtaining the right object for its source, namely the target of $f$.

Then $[g] \circ[f]=\left[s^{-1} t g\right][f]$. So

$$
F\left(s^{-1} u x_{\alpha}\left[s^{-1} t g\right][f]_{x_{\alpha}}\right)=s\left(\left(s^{-1} t g\right)(f)\right)=(t g)(s f)=F(g) F(f) .
$$


It is not hard to see that $F$ is a bijection on objects and an isomorphism of $k$-modules on morphisms.

We end this section with a useful example. Let $\mathcal{B}$ be the Kronecker category with two objects $x$ and $y$, each object having $k$ as endomorphism algebra, and ${ }_{y} \mathcal{B}_{x}=k a \oplus k b$ while ${ }_{x} \mathcal{B}_{y}=0$. Let $G$ be a cyclic group with generator $t$. A $G$ grading of $\mathcal{B}$ is given by $\operatorname{deg} a=1$ and $\operatorname{deg} b=t$. The category $\mathcal{B} \# G$ is the free category of the quiver having vertices $\left(x, t^{i}\right)$ and $\left(y, t^{j}\right)$ and arrows from $\left(x, t^{i}\right)$ to $\left(y, t^{i}\right)$ and to $\left(y, t^{i-1}\right)$ for each $t^{i} \in G$. If $G$ is finite this provides a crown; otherwise the quiver is infinite. The quotient category is clearly the Kronecker category, and the quotient by the action of a proper subgroup provides an intermediate crown.

In case $G$ is free abelian on two generators $s$ and $t$, the grading given by $\operatorname{deg} a=s$ and $\operatorname{deg} b=t$ provides a nonconnected Galois cover through the categorical smash product.

\section{Modules}

Let us recall the following definition:

Definition 4.1. Let $\mathcal{C}$ be a category over a commutative $\operatorname{ring} k$. A left $\mathcal{C}$-module $\mathcal{M}$ is a collection of $k$-modules $\left\{{ }_{x} \mathcal{M}\right\}_{x \in \mathcal{C}_{0}}$ provided with a left action of the $k$-module morphisms of $\mathcal{C}$, given by $k$-module maps ${ }_{y} \mathcal{C}_{x} \otimes_{k}{ }_{x} \mathcal{M} \longrightarrow{ }_{y} \mathcal{M}$ where the image of ${ }_{y} f_{x} \otimes{ }_{x} m$ is denoted ${ }_{y} f_{x}{ }_{x} m$, verifying the usual axioms:

(1) ${ }_{z} f_{y}\left({ }_{y} f_{x}{ }_{x} m\right)=\left({ }_{z} f_{y}{ }_{y} f_{x}\right)_{x} m$;

(2) ${ }_{x} 1_{x}{ }_{x} m={ }_{x} m$.

In other words $\mathcal{M}$ is a covariant functor from $\mathcal{C}$ to the category of $k$-modules. We denote by $\mathcal{C}-$ Mod the category of left $\mathcal{C}$-modules. As is well known and easy to establish the agreement property holds; namely, if $\mathcal{C}$ is a finite number objects category and $a(\mathcal{C})$ is the corresponding algebra of morphisms, $\mathcal{C}$-modules and usual $a(\mathcal{C})$-modules coincide. In particular if $A$ is a $k$-algebra and $\mathcal{C}_{A}$ is the single object category with endomorphism $\operatorname{ring} A$, then $\mathcal{C}_{A}$-modules and $A$-modules are the same.

Let $F: \mathcal{C} \rightarrow \mathcal{B}$ be a functor between categories over $k$. Note that functors do not correspond really to algebra maps. In case $\mathcal{C}$ and $\mathcal{B}$ have a finite number of objects, the corresponding map $a(\mathcal{C}) \rightarrow a(\mathcal{B})$ does not send, in general, $1_{a(\mathcal{C})}$ to $1_{a(\mathcal{B})}$. However the map is additive and multiplicative.

Definition 4.2. Let $F: \mathcal{C} \longrightarrow \mathcal{B}$ be a functor of $k$-categories, and let $\mathcal{N}$ be a $\mathcal{B}$-module. We denote by $F^{*} \mathcal{N}$ the module $\mathcal{N} \circ F$ and by $F^{*}$ the corresponding functor $F^{*}: \mathcal{B}-\operatorname{Mod} \longrightarrow \mathcal{C}-\operatorname{Mod}$.

In other words $F^{*}$ is the restriction functor and $F^{*} \mathcal{N}$ is the $\mathcal{C}$-module given by ${ }_{x}\left(F^{*} \mathcal{N}\right)={ }_{F(x)} \mathcal{N}$ and

$$
\left({ }_{y} c_{x}\right)(F(x) n)=\left({ }_{F(y)} F(c)_{F(x)}\right)\left({ }_{F(x)} n\right) \in{ }_{y}\left(F^{*} \mathcal{N}\right) .
$$

Let $f$ be a natural transformation between modules; then $F^{*}(f)=f$. Note that $F^{*}$ is faithful. In case $F$ is a full functor the functor $F^{*}$ is also full.

In the case of a $G$-category $\mathcal{C}$ over $k$ the group $G$ acts on the category $\mathcal{C}-\operatorname{Mod}$ in the following way: let $\mathcal{M}$ be a $\mathcal{C}$-module and $s \in G$; then ${ }^{s} \mathcal{M}$ is given by

$$
{ }_{x}\left({ }^{s} \mathcal{M}\right)={ }_{s}-1 x \mathcal{M}
$$


and

$$
\left({ }_{y} c_{x}\right)\left({ }_{s^{-1} x} m\right)=\left(s^{-1} c\right) m \in{ }_{s^{-1} y} \mathcal{M}={ }_{y}\left({ }^{s} \mathcal{M}\right) .
$$

Theorem 4.3. Let $F: \mathcal{C} \longrightarrow \mathcal{B}$ be a Galois covering where $\mathcal{C}$ is a free $G$-category over $k$ and $\mathcal{B}=\mathcal{C} / G$. Then $\mathcal{B}-\operatorname{Mod}$ and $(\mathcal{C}-\operatorname{Mod})^{G}$ are isomorphic categories.

Remark 4.4. $(\mathcal{C}-\operatorname{Mod})^{G}$ is a full subcategory of $\mathcal{C}$-Mod provided by fixed objects under the action of $G$, namely modules $\mathcal{M}$ such that ${ }^{s} \mathcal{M}=\mathcal{M}$ for all $s \in G$.

Proof. The image of the functor $F^{*}$ is precisely $(\mathcal{C}-\text { Mod })^{G}$. Indeed

$$
{ }_{x}\left({ }^{s}\left(F^{*} \mathcal{N}\right)\right)={ }_{s^{-1} x}\left(F^{*} \mathcal{N}\right)={ }_{F\left(s^{-1} x\right)} \mathcal{N}={ }_{F(x)} \mathcal{N} .
$$

Note that by definition the functor $F$ of a Galois covering is full.

Next we want to prove that the entire category $\mathcal{C}$-Mod is isomorphic to the category of graded modules over $\mathcal{B}$. Recall that in the case of a Galois covering $F: \mathcal{C} \longrightarrow \mathcal{B}$ (where $\mathcal{C}$ is a free $G$-category over $k$ and $\mathcal{B}=\mathcal{C} / G$ ) we have shown that $\mathcal{B}$ is $G$-graded. We also have shown that $\mathcal{C}=\mathcal{B} \# G$ and we will use this description.

First recall that in case $\mathcal{B}$ is graded, a graded $\mathcal{B}$-module is a $\mathcal{B}$-module $\mathcal{N}$ provided with a direct sum decomposition at each $k$-module, namely

$$
{ }_{x} \mathcal{N}=\bigoplus_{s \in G}{ }_{x} \mathcal{N}^{s} \text { such that }{ }_{y} \mathcal{B}_{x}^{t}{ }_{x} \mathcal{N}^{s} \subset{ }_{y} \mathcal{N}^{t s} .
$$

We denote by $\mathcal{B}-\operatorname{Mod}_{G}$ the category of graded $\mathcal{B}$-modules.

Theorem 4.5. Let $\mathcal{B}$ be a $G$-graded category over $k$ and let $\mathcal{C}=\mathcal{B} \# G$ be its Galois covering. Then $\mathcal{B}-\operatorname{Mod}_{G}$ and $\mathcal{C}-\operatorname{Mod}$ are isomorphic categories.

Proof. Let $\mathcal{N}$ be a graded $\mathcal{B}$-module. We define the $\mathcal{C}$-module $C(\mathcal{N})$ as follows:

$$
{ }_{(x, s)} C(\mathcal{N})={ }_{x} \mathcal{N}^{s^{-1}},
$$

and the action maps

$$
{ }_{(y, t)}(\mathcal{B} \# G)_{(x, s)} \otimes_{k} \quad{ }_{(x, s)} C(\mathcal{N}) \longrightarrow{ }_{(y, t)} C(\mathcal{N})
$$

are given by the maps

$$
{ }_{y} \mathcal{B}_{x}^{t^{-1} s} \otimes_{k}{ }_{x} \mathcal{N}^{s^{-1}} \longrightarrow{ }_{y} \mathcal{N}^{t^{-1}}
$$

Clearly this is an action of $\mathcal{C}$ on $C(\mathcal{N})$ and a map between graded $\mathcal{B}$-modules gives in a functorial way a map between the corresponding $\mathcal{C}$-modules.

Conversely, let $\mathcal{M}$ be a $\mathcal{C}$-module. Let $B(\mathcal{M})$ be the $\mathcal{B}$-module defined by

$$
{ }_{x} B(\mathcal{M})=\bigoplus_{s \in G}(x, s) \mathcal{M} .
$$

The graduation is given by ${ }_{x} B(\mathcal{M})^{s}={ }_{\left(x, s^{-1}\right)} \mathcal{M}$. The action is graded by construction. In order to give the maps

$$
{ }_{y} \mathcal{B}_{x}^{t} \otimes_{k x} B(\mathcal{M})^{s} \longrightarrow{ }_{y} B(\mathcal{M})^{t s},
$$

recall that

Then

$$
{ }_{(y, t)}(\mathcal{B} \# G)_{(x, s)}={ }_{y} \mathcal{B}_{x}^{t^{-1} s}
$$

$$
{ }_{y} \mathcal{B}_{x}{ }^{t}={ }_{\left(y,(t s)^{-1}\right)}(\mathcal{B} \# G)_{\left(x, s^{-1}\right)}
$$


The required maps

$$
{ }_{\left(y,(t s)^{-1}\right)}(\mathcal{B} \# G)_{\left(x, s^{-1}\right)} \otimes_{k}\left(x, s^{-1}\right) \mathcal{M} \longrightarrow{ }_{\left(y,(t s)^{-1}\right)} \mathcal{M}
$$

are obtained through the given action of $\mathcal{B} \# G$ on $\mathcal{M}$.

Clearly the two constructions provide functors which are inverse to each other.

The following result is now clear:

Proposition 4.6. Let $F: \mathcal{C} \longrightarrow \mathcal{B}$ be a Galois covering of categories over $k$ with Galois group $G$. Let $\mathcal{N}$ be a $\mathcal{B}$-module and let $B$ be the functor defined in the proof of the above theorem. Then

$$
B F^{*}(\mathcal{N})=\bigoplus_{s \in G} \mathcal{N}^{s} \text { where } \mathcal{N}^{s}=\mathcal{N} \text { for each } s \in G
$$

The graded action on $\bigoplus_{s \in G} \mathcal{N}^{s}$ is given by ${ }_{y} \mathcal{B}_{x}{ }^{t} \otimes_{k}{ }_{x} \mathcal{N}^{s} \rightarrow{ }_{y} \mathcal{N}^{t}$ using the $\mathcal{B}$-action on $\mathcal{N}$. Ignoring the grading, the $\mathcal{B}$-module $B F^{*}(N)$ is a direct sum of copies of $\mathcal{N}$ indexed by $G$.

\section{ACKNOWLEDGEMENTS}

The authors would like to thank Guillermo Cortiñas and Maria Julia Redondo for useful questions and remarks preluding this work, as well as M. J. Redondo and A. Solotar for a careful reading of a preliminary version of this paper. We also thank the referee, in particular for useful comments concerning previous results.

\section{REFERENCES}

1. Abrams, Gene D. Morita equivalence for rings with local units. Comm. Algebra 11 (1983), 801-837. MR0695890 (85b:16037)

2. Ánh, P. N.; Márki, L. Morita equivalence for rings without identity. Tsukuba J. Math. 11 (1987), 1-16. MR0899719 (88h:16054)

3. Beattie, M. A generalization of the smash product of a graded ring. J. Pure Appl. Algebra 52 (1988), 219-229. MR0952080 (89k:16005)

4. Beattie, M. Duality theorems for rings with actions or coactions. J. Algebra 115 (1988), 303312. MR0943256 (89k:16004)

5. Bongartz, K.; Gabriel, P. Covering spaces in representation-theory. Invent. Math. 65 (1981/82), 331-378. MR0643558 (84i:16030)

6. Cap, A.; Schichl, H.; Vanžura, J. On twisted tensor products of algebras. Comm. Algebra 23 (1995), 4701-4735. MR1352565 (96k:16039)

7. Cibils, C; Redondo, M. J. Cartan-Leray spectral sequence for Galois coverings of categories. J. Algebra 284 (2005), 310-325. MR 2115017

8. Cohen, M.; Montgomery, S. Group-graded rings, smash products, and group actions. Trans. Amer. Math. Soc. 282 (1984), 237-258. MR0728711 (85i:16002)

9. de la Peña, J.A. On the Abelian Galois coverings of an algebra. J. Algebra 102 (1986), 129-134. MR0853234 (87m:16001)

10. del Río, A. Categorical methods in graded ring theory. Publ. Mat. 36 (1992), 489-531. MR:1209821 (94c:16056)

11. Haefner, J. On when a graded ring is graded equivalent to a crossed product. Proc. Amer. Math. Soc. 124 (1996), 1013-1021. MR1301027 (96m:16061)

12. Green, E. L. Graphs with relations, coverings, and group-graded algebras. Trans. Amer. Math. Soc. 279 (1983), 297-310. MR0704617 (85a:16033)

13. Green, E. L.; Marcos, E. N.; Solberg, Ø. Representations and almost split sequences for Hopf algebras. Representation theory of algebras (Cocoyoc, 1994), 237-245, CMS Conf. Proc., 18, Amer. Math. Soc., Providence, RI, (1996). MR1388054 (97i:16033) 
14. Marcos, E. N.; Martins, Ma. I. R.; Martínez-Villa R. Hochschild cohomology of skew group rings and invariants. Cent. Eur. J. Math. 2 (2004), 177-190. MR2113546

15. Menini, C.; Năstăsescu, C. When is R-gr equivalent to the category of modules? J. Pure Appl. Algebra 51 (1988), 277-291. MR0946579 (89f:16002)

16. Năstăsescu, C.; Raianu, Ş.; Van Oystaeyen, F. Modules graded by G-sets. Math. Z. 203 (1990), 605-627. MR1044067 (91h:16071)

17. Năstăsescu C.; Liu, S. X.; Van Oystaeyen, F. Graded modules over G-sets. II. Math. Z. 207 (1991), no. 3, 341-358. MR1115168 (93b:16078)

18. Quinn, D. Group-graded rings and duality. Trans. Amer. Math. Soc. 292 (1985), 155-167. MR 0805958 (87d:16002)

19. Reiten, I.; Riedtmann, C. Skew group algebras in the representation theory of Artin algebras. J. Algebra 92 (1985), 224-282. MR0772481 (86k:16024)

20. Riedtmann, C. Algebren, Darstellungsköcher, Überlagerungen und zurück. Comment. Math. Helv. 55 (1980), 199-224. MR0576602 (82k:16039)

21. Riedtmann, C. MathSciNet review of Green, E. L. Graphs with relations, coverings and groupgraded algebras. Trans. Amer. Math. Soc. 279 (1983), 297-310. MR0704617 (85a:16033)

Institut de Mathématiques et Modélisation de Monpellier, Université de MontpelLier 2, F-34095 Montpellier Cedex 5, France

E-mail address: Claude.Cibils@math.univ-montp2.fr

Departamento de Matemática, Universidade de São Paulo, ime-USP, Caixa Postal 66.281, SÃo PAUlO - SP, 05315-970, Brasil

E-mail address: enmarcos@ime.usp.br 